\title{
Two hits are better than one
}

Studying the mechanisms of tumour immune evasion has led to the identification of several targets for immunotherapy to activate antitumour immune responses. However, multiple immunosuppressive mechanisms may occur at once in a tumour, indicating that combinatorial approaches may be beneficial. Highfill et al. report the possibility of improving responses to immune checkpoint blockade by inhibiting the recruitment of myeloid-derived suppressor cells (MDSCs) into the tumour microenvironment.

Highfill et al. used a syngeneic mouse model of embryonal rhabdomyosarcoma (ERMS), a type of paediatric sarcoma, in which M3-9-M mouse ERMS cells are inoculated into female mice. M3-9-M tumours develop despite activation of peripheral T cells and the authors found evidence of tumour-induced programmed cell death protein 1 (PD1) immune checkpoint signalling, indicating that the tumours can progress by inducing $\mathrm{T}$ cell unresponsiveness through PD1 signalling. Indeed, the treatment of mice with PD1-blocking antibodies at the same time as inoculation prevented tumour development. However, mice with established tumours treated with PD1-blocking antibodies exhibited only a modest (although significant) reduction in tumour growth even though activated $\mathrm{CD} 8^{+} \mathrm{T}$ cells were present in the tumour. Further analyses revealed that the numbers of MDSCs were increased in the spleen and within established M3-9-M tumours, indicating that the MDSCs may suppress the activity of $\mathrm{T}$ cells in response to $\mathrm{PD} 1$ blockade.

To identify the tumour-derived factors responsible for MDSC recruitment, the authors analysed conditioned media from M3-9-M cell culture and found that levels of several cytokines that are known to

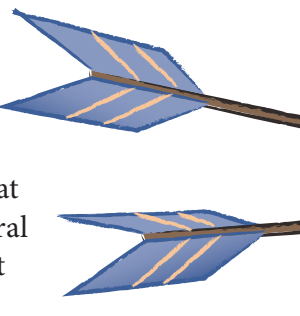
cause MDSC expansion were significantly increased compared with controls. The conditioned media also had high levels of the CXCchemokine receptor 2 (CXCR2) ligands, CXCL1 and CXCL2. CXCR2 was expressed on circulating CD $11 b^{+}$Ly6G ${ }^{\text {hi }}$ cells (markers for granulocytic MDSCs) from mice bearing M3-9-M tumours. Haematopoietic reconstitution with $\mathrm{CxCr}^{-/-}$bone marrow in wild-type mice bearing M3-9-M tumours resulted in a significant reduction in the numbers of CD11b ${ }^{+}$y $6 G^{\text {hi }}$ cells within the tumours, whereas the spleens of these mice had significant increases in the numbers of CD $11 b^{+}$Ly $_{6} \mathrm{G}^{\text {hi }}$ cells. This indicates that although MDSC expansion occurs these cells are no longer recruited to the tumour owing to the lack of CXCR2 expression.

Can CXCR2 be targeted therapeutically to limit MDSC-mediated immunosuppression? Mice bearing established M3-9-M tumours with $\mathrm{CXCr} 2^{-/-}$bone marrow that were treated with PD1-blocking antibodies had reduced tumour growth and increased survival. These tumours also had significantly increased T cell/ MDSC ratios, indicating that failure to recruit MDSCs allows T cell-mediated antitumour immune responses on treatment with anti-PD1 therapy. The treatment of mice bearing established M3-9-M tumours with CXCR2 monoclonal antibodies (mAbs) also significantly reduced numbers of
CD $11 b^{+}{ }^{+}$6y $6 G^{\text {h }}$ cells within the tumours (but not in the spleens).

Furthermore,

combining CXCR2 mAbs with PD1-blocking antibodies significantly reduced tumour growth and significantly improved the survival of mice bearing established M3-9-M tumours, more so than either treatment alone.

Analysis of the serum from 53 patients with metastatic paediatric sarcoma revealed that levels of CXCL1 and CXCL8, the major ligands for CXCR2 in humans, were significantly higher compared with controls and high levels of CXCL8 inversely correlated with survival. This indicates that circulating CXCR2 ligands may also recruit CXCR2 ${ }^{+}$MDSCs in patients with sarcoma, suggesting that combined anti-CXCR2 and anti-PD1 therapy could be beneficial to these patients. However, it is important to note that MDSCs are not well characterized in humans and so further work is required to clarify the phenotype of human MDSCs before they can be therapeutically targeted in patients.

Gemma K. Alderton

ORIGINAL RESEARCH PAPER Highfill, S. L. et al. Disruption of CXCR2-mediated MDSC tumor trafficking enhances anti-PD1 efficacy. Sci. Transl Med. 6, 237 ra67 (2014) 